\title{
Hybrid approach to black hole perturbations from extended matter sources
}

\author{
Valeria Ferrari, ${ }^{1}$ Leonardo Gualtieri, ${ }^{1}$ and Luciano Rezzolla ${ }^{2,3,4}$ \\ ${ }^{1}$ Dipartimento di Fisica, Università di Roma "La Sapienza” and INFN Rome, Italy \\ ${ }^{2}$ Max-Planck-Institut für Gravitationsphysik, Albert-Einstein-Institut, Potsdam Germany \\ ${ }^{3}$ SISSA, International School for Advanced Studies and INFN Trieste, Italy \\ ${ }^{4}$ Department of Physics, Louisiana State University, Baton Rouge, Lousiana USA
}

(Received 5 May 2006; published 20 June 2006)

\begin{abstract}
We present a new method for the calculation of black hole perturbations induced by extended sources in which the solution of the nonlinear hydrodynamics equations is coupled to a perturbative method based on Regge-Wheeler/Zerilli and Bardeen-Press-Teukolsky equations when these are solved in the frequency domain. In contrast to alternative methods in the time domain which may be unstable for rotating black hole spacetimes, this approach is expected to be stable as long as an accurate evolution of the matter sources is possible. Hence, it could be used under generic conditions and also with sources coming from three-dimensional numerical relativity codes. As an application of this method we compute the gravitational radiation from an oscillating high-density torus orbiting around a Schwarzschild black hole and show that our method is remarkably accurate, capturing both the basic quadrupolar emission of the torus and the excited emission of the black hole.
\end{abstract}

DOI: 10.1103/PhysRevD.73.124028

PACS numbers: 04.30.Db, 04.40.Dg, 95.30.Lz, 97.60.Lf

\section{INTRODUCTION}

It is well-known that matter falling into a black hole, or orbiting around it, is a source of gravitational radiation. The detection of these waves, and of the imprint they carry on the excitation of the black hole quasinormal modes (QNM), would provide an invaluable test of general relativity in the strong-field regime. This process can be studied using the theory of black hole perturbations, the origin of which dates back to 1957 when Regge and Wheeler [1] showed that the axial perturbations of a Schwarzschild black hole can be studied by solving a radial equation in the frequency domain, the angular part of the perturbation being known in terms of a suitable expansion in tensor spherical harmonics.

Among the many dedicated to this subject, two papers can be considered as milestones in the theory of black hole perturbations. The first one is by F. Zerilli [2], who derived the radial equation governing the polar perturbations of a Schwarzschild black hole, thus completing the work of Regge and Wheeler (see [3] for a recent review). The second one is by S. Teukolsky [4], who achieved the formidable task of reducing the equations for the perturbations of a Kerr black hole to a master radial equation. Both the Zerilli and the Teukolsky equations were derived in the frequency domain.

The theory of black hole perturbations has been developed largely to study the stability properties of black holes and to determine the gravitational signals emitted by point particles moving near black holes on different orbits (see for instance [5] and references therein). In addition, it has been used to evaluate back-reaction effects on the orbital motion of a point particle moving around a nonrotating black hole [6].

Although it clearly represents only a toy-model, the point-particle approximation has been very useful to study a number of phenomena that occur in the neighborhood of black holes and it has also been applied to study stellar perturbations $[7,8]$. However, if one wants to investigate realistic astrophysical processes, in which finite-size effects and internal dynamics need to be properly taken into account, the point-particle approximation is certainly too crude. Therefore, several attempts have been made towards a more realistic description. In Ref. [9], for instance, the point particle orbiting a black hole was treated as having a spin angular momentum, while in Ref. [10] the infalling matter was assumed to be a finite size sphere of dust. Furthermore, in Ref. [11] a semianalytic method was developed to study the radial infall of a shell of dust; while this latter approach has the advantage of being easily generalizable to sources with arbitrary shapes, it has the serious drawback that it can be applied only to sources in which the matter configurations are freely falling onto the black hole. It is worth stressing that in all cases mentioned above the black hole is assumed to be nonrotating.

Alternative and more general approaches to treat extended sources have recently been developed by Papadopulous and Font [12] and by Nagar et al. [13], by combining perturbative approaches with fully multidimensional hydrodynamical calculations. In Ref. [13], in particular, the polar perturbations of a Schwarzschild black hole induced by an oscillating, thick accretion disk (a torus) have been studied by solving the inhomogenous Zerilli equation in the time domain. The source terms were computed self-consistently by using the results of two-dimensional (2D) simulations in which the relativistic hydrodynamical equations were solved for a torus oscillating in the Schwarzschild, unperturbed background. The purpose of that work was to compare the gravitational signal found by solving the Zerilli equation, with the one 
found using the standard quadrupole formalism applied to the oscillating torus.

In this paper we develop a new approach to study the perturbations of a Schwarzschild black hole excited by extended matter sources, by solving the inhomogeneous Bardeen-Press-Teukolsky (BPT) equation [14,15] in the frequency domain. Also in this case the source for the perturbative equations is the result of independent 2D hydrodynamical simulations in the time domain. Because of the combined use of both time and frequency domains approaches, we refer to this as to a hybrid approach.

There are at least three good reasons why our hybrid approach may be preferable over a pure time-domain approach as the one used in [13]. Firstly, the ReggeWheeler and the Zerilli equations (hereafter RWZ equations) cannot be generalized to rotating black holes, whereas the BPT equation admits such a generalization, i.e., the Teukolsky equation. (We recall that the Teukolsky equation is not separable in the time domain because the eigenvalues of the angular functions are frequency dependent; this is not the case in the frequency domain where the equation is indeed separable.) Secondly, although the BPT equation is intrinsically unstable when integrated in the time domain, its integration does not offer any numerical difficulty when performed in the frequency domain. Thirdly, it is a common experience that by removing the error in the time integration of a system oscillating around its equilibrium state, a frequency-domain approach improves the overall accuracy. The work presented here serves therefore to develop a new procedure for the solution of the BPT equation with extended matter sources, whose generalization will allow to reach a long-standing goal: i.e., the study of the gravitational-wave emission from an extended source perturbing a rotating black hole.

While the mathematical apparatus behind our hybrid approach is well-know, our numerical implementation is more innovative and to test it against an astrophysically realistic and computationally complex configuration we have here also considered the gravitational-wave emission from a high-density torus oscillating in its orbital motion around a Schwarzschild black hole. This is the same source considered in Ref. [13] and thus offers the possibility of comparing for the same test case our hybrid approach with one which is instead in the time domain only.

To this scope, we recall that the possibility that extremely dense and massive accretion tori form around a black hole has been considered in recent years, initially as a possible explanation of $\gamma$-ray bursts [16]. In these systems, a torus of mass $M_{t} \sim 0.1-1 M_{\odot}$ and mean density as high as $\sim 10^{11}-10^{12} \mathrm{~g} / \mathrm{cm}^{3}$, forms around a black hole of a few solar masses. The torus can be located within a few tens of $\mathrm{km}$ from the horizon and could also be subject to a dynamical instability which would induce its accretion onto the black hole on a dynamical timescale (see $[17,18]$ and references therein). High-density tori could be generated in many astrophysical scenarios (see $[16,18]$ for a general overview): in the coalescence of black holeneutron star or neutron star binaries, as shown by several numerical simulations [19] in the collapse to a black hole of a rapidly rotating supramassive neutron star [20,21], as a byproduct of collapsars, that is, massive stars in which iron core-collapse does not produce an explosion, but forms a black hole [22]. As discussed in [18], these systems can be created with event rates comparable to that of corecollapse supernovae. In addition, because the tori have an intrinsically large quadrupole moment, even smallamplitude oscillations would produce gravitational signals that may be detectable by ground based interferometers Virgo and LIGO [18]. As a result, the calculation of the gravitational waves from these tori does not only represent a useful testbed for our novel approach, but it also offers the opportunity for a more accurate calculation of the emission from a realistic and intense source of gravitational radiation.

The gravitational radiation emitted by a torus oscillating around a black hole was first computed in Ref. [18] within the Newtonian quadrupole formalism. However, the quadrupole formalism may not be able to describe a system with such a high energy density, and located so close to a black hole horizon with sufficient accuracy. In particular, the possible excitation of the black hole QNM cannot be revealed by the quadrupole approach. The same system has been studied in [13], where the perturbed equations have been integrated in the time domain and no excitation of the black hole quasinormal modes (QNMs) was found. Conversely, as we will later discuss, our hybrid method applied to the same system allows to catch the excitation of the black hole quasinormal modes, though the peak they produce in the energy spectrum is much smaller than that corresponding to the torus oscillation.

The plan of the paper is as follows: in Sec. II we review the main equations which describe the perturbations of a Schwarzschild black hole in the RWZ and in the BPT approaches when these are expressed in the frequency domain. Furthermore, we also recall the expression of the gravitational energy flux computed within the Newtonian quadrupole formalism. In Sec. III we present the analytical and numerical setup of our approach for the solution of both the perturbative and general relativistic hydrodynamics equations. Finally, in Sec. IV we discuss the results of the numerical integrations for the representative case of an oscillating torus, while in Sec. V we draw our conclusions. A number of Appendices contain details on the form of the source functions employed for the solution of the perturbative equations.

\section{A BRIEF REVIEW OF BLACK HOLE PERTURBATIONS IN THE FREQUENCY DOMAIN}

In what follows we briefly summarize the relevant equations needed to describe the perturbations of a 
Schwarzschild spacetime in the RWZ and in the BPT formulations, as they will later be used in our hybrid approach. We will also write the expression of the energy flux computed within the Newtonian quadrupole approximation since it will be used for comparison with the corresponding quantities computed within the perturbative approaches.

\section{A. The RWZ approach}

In the RWZ approach [1,2], the equations describing the radial behavior of the perturbations of a Schwarzschild black hole are reduced to two wave equations after expanding all perturbed tensors in tensorial spherical harmonics and after separating the radial from the angular part. In particular, they are expressed for two suitable combinations of the components of the perturbed metric tensor and which are referred to as the Zerilli function, $\tilde{Z}_{\ell m}^{(+)}$, and the Regge-Wheeler function, $\tilde{Z}_{\ell m}^{(-)}$, respectively. Hereafter we will indicate with a "tilde" all the perturbative variables that have a time dependence which is not explicitly indicated and with $\ell, m$ the indices of the spherical harmonic decomposition.

Since the system we will consider, namely, the oscillating torus perturbing the spherically symmetric black hole spacetime, is axially symmetric, the perturbations are also axially symmetric, and hence in what follows we will consider $m=0$, with the Zerilli and Regge-Wheeler functions expressed as $\tilde{Z}_{\ell}^{(+)}(r, t), \tilde{Z}_{\ell}^{(-)}(r, t)$, with $\ell \geq 2$.

Their Fourier transforms are simply given by

$$
Z_{\ell}^{( \pm)}(r, \omega) \equiv \frac{1}{2 \pi} \int e^{\mathrm{i} \omega t} \tilde{Z}_{\ell}^{( \pm)}(r, t) d t,
$$

and satisfy the inhomogeneous wave equations

$$
\mathbb{L}^{( \pm)} Z_{\ell}^{( \pm)}=S_{\ell}^{( \pm)},
$$

where the upper index "+" refers to the Zerilli equation, the "-" one to the Regge-Wheeler equation, and the symbol $\mathbb{L}^{( \pm)}$is just a shorthand for the differential operator

$$
\mathbb{L}^{( \pm)} \equiv \partial_{r_{*}}^{2}+\omega^{2}-V^{( \pm)} .
$$

Note that in order to provide mathematically consistent boundary conditions at the event horizon, the latter is moved to negative spatial infinity in terms of the "tortoise" radial coordinate $r_{*} \equiv r+2 M \ln (r / 2 M-1)$, and that the Zerilli and Regge-Wheeler potentials in Eq. (2.2) have explicit expressions given, respectively, by

$$
\begin{aligned}
V^{(+)} \equiv & \frac{2(r-2 M)}{r^{4}(\Lambda r+3 M)^{2}}\left[\Lambda^{2}(\Lambda+1) r^{3}+3 M \Lambda^{2} r^{2}\right. \\
& \left.+9 M^{2} \Lambda r+9 M^{2}\right] \\
& V^{(-)} \equiv \frac{2(r-2 M)}{r^{4}}[(\Lambda+1) r-3 M] .
\end{aligned}
$$

Here $\Lambda \equiv(\ell-1)(\ell+2) / 2$, while $S_{\ell}^{( \pm)}(r, \omega)$ are suitable combinations of the components of the stress-energy tensor of the extended matter source, after having been expanded in tensor spherical harmonics and Fourier transformed in time. Explicit expressions for the source functions $S_{\ell}^{( \pm)}(r, \omega)$ can be found in Appendix A 1.

Equations (2.2) can to be solved numerically by imposing outgoing boundary conditions at spatial infinity (i.e., $\left.r, r_{*} \rightarrow \infty\right)$, and ingoing boundary conditions at the event horizon (i.e., $r=2 M, r_{*} \rightarrow-\infty$ ). The energy spectrum of the gravitational radiation measured by a distant observer and integrated over the solid angle, can then be expressed in terms of the asymptotic amplitude of $Z_{\ell}^{(+)}$and $Z_{\ell}^{(-)}$as

$$
\begin{aligned}
\frac{d E^{\mathrm{ZRW}}}{d \omega} & =\left.\sum_{l=2}^{\infty} \frac{d E_{\ell}}{d \omega}\right|_{r, r_{*} \rightarrow \infty} \\
& =\sum_{l=2}^{\infty} \frac{1}{8} \frac{(\ell+2) !}{(\ell-2) !}\left[\omega^{2}\left(Z_{\ell}^{(+)}\right)^{2}+\left(Z_{\ell}^{(-)}\right)^{2}\right] .
\end{aligned}
$$

\section{B. The BPT approach}

In the BPT approach $[14,15]$, the perturbations of the Weyl scalar $\delta \Psi_{4}$, which describes the outgoing gravitational radiation, are expanded in spin-weighted spherical harmonics ${ }_{-2} S_{\ell m}^{*}$ and integrated over the solid angle $d \Omega=$ $\sin \theta d \theta d \phi$

$$
\tilde{\Psi}_{\ell m}(r, t)=\int d \Omega_{-2} S_{\ell m}^{*}(\vartheta, \phi) r^{4} \delta \tilde{\Psi}_{4}(t, r, \vartheta, \phi) .
$$

Also in this case, being the perturbing stress-energy tensor axially symmetric the only nonvanishing components are those with $m=0$ and hence perturbed Einstein equations take the form of inhomogeneous wave equations

$$
\mathbb{L}^{\mathrm{BPT}} \Psi_{\ell}(r, \omega)=-S_{\ell}^{\mathrm{BPT}}(r, \omega),
$$

where $\Psi_{\ell}(r, \omega)$ is the Fourier transform of $\tilde{\Psi}_{\ell}(r, t)$, the symbol $\mathbb{L}^{\mathrm{BPT}}$ is just a shorthand for the differential operator,

$$
\llbracket^{\mathrm{BPT}} \equiv \Delta^{2} \partial_{r} \frac{1}{\Delta} \partial_{r}+U(r),
$$

with $\Delta \equiv r(r-2 M)$ and the potential $U$ having explicit form

$$
U \equiv \frac{r^{4} \omega^{2}+4 \mathrm{i}(r-M) r^{2} \omega}{\Delta}-8 \mathrm{i} \omega r-2 \Lambda .
$$

As for the RWZ equation, the source terms $S_{\ell}^{\mathrm{BPT}}(r, \omega)$ are suitable combinations of components of the stress-energy tensor after having been expanded in spin-weighted spherical harmonics and Fourier transformed. Explicit expressions for the source functions $S_{\ell}^{( \pm)}(r, \omega)$ can be found in Appendix A 2. 
Equation (2.8) can be integrated numerically imposing the same boundary conditions discussed for the RWZ equation. In this case, the energy spectrum of the gravitational radiation collected by a distant observer and integrated over the solid angle is expressed as

$$
\frac{d E^{\mathrm{BPT}}}{d \omega}=\left.\sum_{\ell=2}^{\infty} \frac{d E_{\ell}}{d \omega}\right|_{r_{*} \rightarrow \infty}=\sum_{\ell=2}^{\infty} \frac{8 \pi^{2}}{\omega^{2}}\left|\frac{\Psi_{\ell}}{r^{3}}\right|^{2} .
$$

\section{The Newtonian quadrupole approximation}

The Newtonian quadrupole approximation is based on the assumptions that the gravitational field is weak, that the velocity of matter in the source is much smaller than the speed of light, and that the source itself is small compared to the characteristic wavelength of the emitted gravitational radiation. In this approach, then, the amplitude of the gravitational wave is estimated in terms of the quadrupole tensor associated to the source

$$
\tilde{q}_{i j} \equiv \int_{V} \tilde{T}^{00}(t, \vec{x}) x^{i} x^{j} d^{3} x \quad(i, j=1,2,3) .
$$

where $\boldsymbol{T}$ is the stress-energy tensor of the matter source. The resulting gravitational-wave energy spectrum is then given by

$$
\frac{d E^{\text {quad }}}{d \omega}=\frac{4 \pi}{5} \omega^{6} \sum_{i j}\left|Q_{i j}(\omega) Q_{i j}(\omega)\right|
$$

where $Q_{i j}(\omega)$ is the Fourier transform of the traceless quadrupole tensor $\tilde{Q}_{i j}$

$$
\tilde{Q}_{i j} \equiv \tilde{q}_{i j}-\frac{1}{3} \delta_{i j} \sum_{k l} \delta_{k l} \tilde{q}_{k l}
$$

As we will comment more extensively in Sec. IV, we will compare expressions (2.6), (2.11), and (2.13) for the gravitational emission coming from an oscillating torus and point out the respective advantages and disadvantages.

\section{THE HYBRID APPROACH}

We will now give a general and brief account of our hybrid approach, sketching its most relevant parts and its numerical implementation. We will first discuss how to construct the components of the stress-energy tensor, needed as source terms in the perturbative equations, through the solution of the 2D hydrodynamic equations in a Schwarzschild background. We will then illustrate how to find the solution of the RWZ and BPT equations once the sources are known in the frequency domain.

\section{A. Computing the sources from the hydrodynamic equations}

As mentioned in the Introduction, any realistic and extended matter source whose dynamics goes beyond the elementary free-fall from infinity, will need to be described through the solution of the general relativistic hydrodynamics equations on the background black hole spacetime. In particular, this is accomplished by computing at the different spacetime points covered by a numerical grid, the components of the stress-energy tensor $T_{\mu \nu}(t, r, \theta)$ necessary for the calculation of the sources $\tilde{S}_{\ell}^{( \pm)}$and $\tilde{S}_{\ell}^{\mathrm{BPT}}$.

For a matter source represented by a perfect fluid with four-velocity $\boldsymbol{u}$ and described by the stress-energy tensor

$$
T_{\mu \nu} \equiv(e+p) u_{\mu} u_{\nu}+p g_{\mu \nu}=\rho h u_{\mu} u_{\nu}+p g_{\mu \nu},
$$

where $g_{\mu \nu}$ are the coefficients of the metric which we choose to be Schwarzschild. Here, $e, p, \rho$, and $h=(e+$ $p) / \rho$ are the proper energy density, the isotropic pressure, the rest-mass density, and the specific enthalpy, respectively. In practice, all of these quantities are computed by the solution of the conservation equations for the stressenergy tensor and baryon number density

$$
\begin{gathered}
\nabla_{\mu} T^{\mu \nu}=0, \\
\nabla_{\mu}\left(\rho u^{\mu}\right)=0,
\end{gathered}
$$

where $\nabla$ indicates the covariant derivative in the background Schwarzschild spacetime, together with an equation of state (EOS) relating the pressure to other thermodynamical quantities. For simplicity we will hereafter model the fluid as ideal with a polytropic $p=\kappa \rho^{\gamma}=$ $\rho \epsilon(\gamma-1)$, where $\epsilon=e / \rho-1$ is the specific internal energy, $\kappa$ is the polytropic constant and $\gamma$ is the adiabatic index.

In order to preserve their conservative nature, we cast Eqs. (3.2) and (3.3) in the form of a flux-conservative hyperbolic system after introducing suitable "conserved" variables rather than in terms of the ordinary fluid, or "primitive", variables. In this case, Eqs. (3.2) and (3.3) assume the form [23]

$$
\frac{\partial \mathbf{U}(\mathbf{w})}{\partial t}+\frac{\partial\left[\sqrt{-g_{00}} \mathbf{F}^{r}(\mathbf{w})\right]}{\partial r}+\frac{\partial\left[\sqrt{-g_{00}} \mathbf{F}^{\theta}(\mathbf{w})\right]}{\partial \theta}=\mathbf{S}(\mathbf{w}),
$$

where $\mathbf{U}(\mathbf{w})=\left(D, S_{r}, S_{\theta}, S_{\phi}\right), \mathbf{F}^{i}$ and $\mathbf{S}$ are the statevector, the fluxes and the sources of the evolved quantities, respectively, (see [17] for the explicit expressions for $\mathbf{F}^{i}$ and $\mathbf{S}$ in a Schwarzschild spacetime). The following set of equations

$$
D \equiv \rho \Gamma, \quad S_{j} \equiv \rho h \Gamma^{2} v_{j},
$$

together with the ideal-fluid EOS provide the relation between the conserved and primitive variables in the vector $\mathbf{w}=\left(\rho, v_{i}, \epsilon\right)$. Here $\Gamma \equiv \alpha u^{t}=\left(1-v^{2}\right)^{-1 / 2}$, where $v^{2} \equiv$ $\gamma_{i j} v^{i} v^{j}$ is the Lorentz factor measured by a local static 
observer. Note that the covariant components of the threevelocity are defined in terms of the spatial 3-metric $\gamma_{i j}$ to be $v_{i}=\gamma_{i j} v^{j}$, where $v^{i}=u^{i} / \alpha u^{t}$. (Although in axisymmetry, we evolve also the azimuthal component of the equations of motion, so that the index $j$ takes the values $j=r, \theta, \phi$.)

The numerical code used in our computations is the same used in ref. [18] and it performs the numerical integration of system (3.4) using upwind high-resolution shock-capturing (HRSC) schemes based on approximate Riemann solvers. Exploiting the flux-conservative form of Eqs. (3.4), the time evolution of the discretized data from a time-level $n$ to the subsequent one $n+1$ is performed according to the following scheme

$$
\begin{aligned}
\mathbf{U}_{i, j}^{n+1}= & \mathbf{U}_{i, j}^{n}-\frac{\Delta t}{\Delta r}\left(\hat{\mathbf{F}}_{i+1 / 2, j}^{r}-\hat{\mathbf{F}}_{i-1 / 2, j}^{r}\right) \\
& -\frac{\Delta t}{\Delta \theta}\left(\hat{\mathbf{F}}_{i, j+1 / 2}^{\theta}-\hat{\mathbf{F}}_{i, j-1 / 2}^{\theta}\right)+\Delta t \mathbf{S}_{i, j},
\end{aligned}
$$

where the subscripts $i, j$ refer to spatial $(r, \theta)$ grid points, so that $\mathbf{U}_{i, j}^{n} \equiv \mathbf{U}\left(r_{i}, \theta_{j}, t^{n}\right)$. The intercell numerical fluxes, $\hat{\mathbf{F}}_{i \pm 1 / 2, j}^{r}$ and $\hat{\mathbf{F}}_{i, j \pm 1 / 2}^{\theta}$, are computed using Marquina's approximate Riemann solver [18]. A piecewise-linear cell reconstruction procedure provides second-order accuracy in space, while the same order in time is obtained with a conservative two-step second-order Runge-Kutta scheme applied to the above time update.

Our computational grid consists of $N_{r} \times N_{\theta}=250 \times$ 84 zones in the radial and angular directions, respectively, covering a computational domain extending from $r_{\text {min }}=$ 2.1 to $r_{\max }=30$ and from 0 to $\pi$. The radial grid is logarithmically spaced in terms of the tortoise coordinate with the maximum radial resolution at the innermost grid being $\Delta r=6 \times 10^{-4}$. As in Ref. [18], we use a finer angular grid in the regions that are usually within the torus and a much coarser one outside. The boundary conditions adopted, the treatment of the vacuum region outside the torus with a low density atmosphere, and the procedure for recovering physical variables from the conserved quantities $D$ and $S_{i}$ are the same as those used in Ref. [18]. The interested reader is referred to that work for further details.

\section{B. Solution of the RWZ and BPT equations}

Before solving Eq. (2.2) with ingoing wave boundary condition at the horizon and outgoing wave boundary condition at infinity, we determine the solution of the corresponding homogeneous equation

$$
\mathbb{\Perp}^{( \pm)} Z_{\ell}^{( \pm)}=0
$$

for an assigned value of $\omega$, finding two particular solutions, $Z_{\ell}^{( \pm) \text {in }}, Z_{\ell}^{( \pm) \text {out }}$, which satisfy, respectively, ingoing wave boundary conditions at the horizon

$$
Z_{\ell}^{( \pm) \text {in }}\left(r_{*} \rightarrow-\infty, \omega\right)=e^{-\mathrm{i} \omega r_{*}},
$$

and outgoing wave boundary conditions at infinity

$$
Z_{\ell}^{( \pm) \text {out }}\left(r_{*} \rightarrow \infty, \omega\right)=e^{\mathrm{i} \omega r_{*}} .
$$

Since they are independent solutions, their Wronskian

$$
W_{*} \equiv Z_{\ell}^{( \pm) \text {out }}\left(\frac{\partial Z_{\ell}^{( \pm) \text {in }}}{\partial r_{*}}\right)-\left(\frac{\partial Z_{\ell}^{( \pm) \text {out }}}{\partial r_{*}}\right) Z_{\ell}^{( \pm) \text {in }}
$$

is constant. The solution of the inhomogeneous Eqs. (2.2), $Z_{\ell}^{( \pm)}$, is then found as a convolution integral of the source with $Z_{\ell}^{( \pm) \text {in }}$ and $Z_{\ell}^{( \pm) \text {out }}$

$$
\begin{aligned}
Z_{\ell}^{( \pm)}(r, \omega)= & \frac{Z_{\ell}^{( \pm) \text {out }}}{W_{*}} \int_{2 M}^{r} d r^{\prime} \frac{Z_{\ell}^{( \pm) \text {in }} S_{\ell}^{( \pm)} r^{\prime 2}}{\Delta} \\
& +\frac{Z_{\ell}^{( \pm) \text {out }}}{W_{*}} \int_{r}^{\infty} d r^{\prime} \frac{Z_{\ell}^{( \pm) \text {out }} S_{\ell}^{( \pm)} r^{\prime 2}}{\Delta} .
\end{aligned}
$$

At spatial infinity the Zerilli function can be written as [24]

$$
Z_{\ell}^{( \pm)}(r, \omega) \rightarrow \mathcal{A}_{\ell}^{( \pm)}(\omega) e^{i \omega r},
$$

where

$$
\mathcal{A}_{\ell}^{( \pm)}(\omega) \equiv \frac{1}{W_{*}} \int_{2 M}^{\infty} d r \frac{Z_{\ell}^{( \pm) i n} S_{\ell}^{( \pm)} r^{2}}{\Delta} .
$$

In this way, the functions $\mathcal{A}_{\ell}^{(+)}$and $\mathcal{A}_{\ell}^{(-)}$represent the amplitudes of the gravitational radiation with polar and axial parity and, as expressed by (2.6), they both contribute to the gravitational spectrum

$$
\frac{d E^{\mathrm{RWZ}}}{d \omega}=\sum_{\ell=2}^{\infty} \frac{1}{8} \frac{(\ell+2) !}{(\ell-2) !}\left[\omega^{2}\left(\mathcal{A}_{\ell}^{(+)}\right)^{2}+\left(\mathcal{A}_{\ell}^{(-)}\right)^{2}\right] .
$$

As done for the RWZ equations, also for the BPT Eq. (2.8) we first solve the corresponding homogeneous equation

$$
\llbracket^{\mathrm{BPT}} \Psi_{\ell}(r, \omega)=0
$$

for an assigned value of $\omega$ and find two independent solutions which satisfy, respectively, the condition of a pure ingoing wave at the black hole horizon and of a pure outgoing wave at infinity

$$
\begin{gathered}
\Psi_{\ell}^{\text {in }}\left(r_{*} \rightarrow-\infty, \omega\right)=\Delta^{2} e^{-\mathrm{i} \omega r_{*}}, \\
\Psi_{\ell}^{\text {out }}\left(r_{*} \rightarrow \infty, \omega\right)=r^{3} e^{\mathrm{i} \omega r_{*}},
\end{gathered}
$$

where the correcting factors $\Delta$ and $r^{3}$ in (3.16) and (3.17) are the result of the asymptotic expansion of the BPT equation $[14,15]$.

The solution $\Psi_{\ell}(r, \omega)$ of the inhomogeneous Eq. (2.8) has a form analogous to the one for the RWZ Eqs. (3.11) and also in this case for $r_{*} \rightarrow \infty$ it can be expressed as

$$
\Psi_{\ell}\left(r_{*}, \omega\right) \rightarrow \mathcal{A}_{\ell}^{\mathrm{BPT}}(\omega) r^{3} e^{\mathrm{i} \omega r_{*}},
$$

with 


$$
\mathcal{A}_{\ell}^{\mathrm{BPT}}(\omega)=-\frac{1}{W} \int_{2 M}^{\infty} d r^{\prime} \frac{\Psi_{\ell}^{\mathrm{in}} S_{\ell}^{\mathrm{BPT}}(r, \omega)}{\Delta^{2}}
$$

where the Wronskian is now defined as

$$
W \equiv \frac{1}{\Delta}\left[\Psi_{\ell}^{\text {in }}\left(\frac{\partial \Psi_{\ell}^{\text {out }}}{\partial r}\right)-\left(\frac{\partial \Psi_{\ell}^{\text {in }}}{\partial r}\right) \Psi_{\ell}^{\text {out }}\right] .
$$

Also in this case, the complex amplitude $\mathcal{A}_{\ell}^{\mathrm{BPT}}$ describes the gravitational-wave amplitudes with polar and axial parity, so that the gravitational spectrum (2.11) becomes

$$
\frac{d E^{\mathrm{BPT}}}{d \omega}=\sum_{\ell=2}^{\infty} \frac{8 \pi^{2}}{\omega^{2}}\left|\mathcal{A}_{\ell}^{\mathrm{BPT}}\right|^{2} .
$$

It is important to note that the solutions $\Psi_{\ell}^{\text {in/out }}$ of the homogeneous Eqs. (3.16) and (3.17), can be expressed in terms of the solutions $Z_{\ell}^{(-) \text {in/out }}$ of the corresponding homogeneous RWZ Eqs. (3.7) through the Chandrasekhar transformation [25]

$$
\begin{aligned}
\Psi_{\ell}^{\text {in/out }}= & \frac{r^{3}}{8 \omega} \sqrt{\frac{(\ell+2) !}{(\ell-2) !}}\left[V^{(-)} Z_{\ell}^{(-) \text {in } / \text { out }}+2\left(\frac{r-3 M}{r^{2}}+\mathrm{i} \omega\right)\right. \\
& \left.\times\left(\frac{\Delta}{r^{2}} Z_{\ell, r}^{(-) \text {in } / \text { out }}+\mathrm{i} \omega Z_{\ell}^{(-) \text {in } / \text { out }}\right)\right] .
\end{aligned}
$$

In what follows we will use this transformation to avoid the resolution of (3.15).

\section{Combining the two approaches}

Having described the distinct approaches for the calculation of the sources in the time domain and the solution of the perturbative equations in the frequency domain, we now discuss how to combine the two methods within our hybrid approach. Since the methodology applies unchanged whether we are considering the solution of the RWZ or of the BPT equations, we will drop this distinction in what follows.

Assume therefore that the solution of the hydrodynamical Eqs. (3.2) and (3.3) as illustrated in Sec. III A has provided the components of the stress-energy tensor $T_{\mu \nu}(t, r, \theta)$ for all the spacetime events that are relevant. The first step for the effective calculation of the sources consists then in the removal of the angular dependence. This is done by calculating for the different values of $\ell$, the integrals over the 2-sphere of the stress-energy tensor components, as given in Eqs. (A11)-(A17) and (A33)(A35) to compute the following quantities

$$
\begin{array}{cccccc}
\tilde{A}_{\ell}, & \tilde{A}_{\ell}^{(1)}, & \tilde{B}_{\ell}^{(0)}, & \tilde{B}_{\ell}, & \tilde{Q}_{\ell}, & \tilde{F}_{\ell}, \\
\tilde{D}_{\ell}, & \tilde{T}_{(n)(n) \ell}, & \tilde{T}_{(n)(\bar{m}) \ell}, & \tilde{T}_{(\bar{m})(n \bar{m}) \ell} . &
\end{array}
$$

As a result, all of the above quantities are functions of $(t, r)$ only and are evaluated at the discrete spacetime points $r_{i}$, $t_{j}$, where $r_{i}$ coincide with the radial gridpoints of the $2 \mathrm{D}$ code. The time levels $t_{j}=j \Delta t \in[0, T]$, on the other hand, are chosen so that $T \gg \tau$, where $\tau$ is the typical timescale for the problem (e.g., the torus oscillation timescale for the problem at hand and $T / \tau \simeq 100$ ), and $\Delta t \ll \tau$ (e.g., $\Delta t / \tau \simeq 10^{-2}-10^{-3}$ ). We note that in general the solution of the hydrodynamical equations does not take place on equally spaced spacelike hypersurfaces since the time step is automatically adjusted on the basis of the dynamics of the matter source. As a result, the evaluation of the functions (3.23) on the equally spaced time levels $t_{j}$ requires in general an interpolation process.

Once the interpolated timeseries for the functions (3.23) have been constructed at each gridpoint $r_{i}$, these are Fourier transformed yielding the corresponding frequency-domain functions

$\begin{array}{lrrrrr}A_{\ell}, & A_{\ell}^{(1)}, \quad B_{\ell}^{(0)}, & B_{\ell}, & Q_{\ell}, & F_{\ell}, & D_{\ell}, \\ T_{(n)(n) \ell}, & T_{(n)(\bar{m}) \ell}, & T_{(\bar{m})(n \bar{m}) \ell} & & & \text { (3.24) }\end{array}$

which are evaluated at the points $\left(r_{i}, \omega_{j}\right)$, with $\omega_{j}=$ $j \Delta \omega=j 2 \pi / T$. The functions (3.24) are then suitably combined as in Eqs. (A20), (A21), and (A32) to provide the compact forms for the source terms in the frequency domain, $S_{\ell}^{( \pm)}\left(r_{i}, \omega_{j}\right), S_{\ell}^{\mathrm{BPT}}\left(r_{i}, \omega_{j}\right)$.

The next step consists of integrating the homogeneous Eqs. (3.7) to find the two independent solutions (3.8) and (3.9). The numerical solution is found using a $4^{\text {th }}$-order Runge-Kutta integrator with adaptive stepsize and with boundary conditions given by expressions (3.8) and (3.9). Using the newly computed RWZ solutions $Z_{\ell}^{( \pm) \text {in/out }}\left(r_{i}, \omega_{j}\right)$ and the transformation (3.22), it is possible to also compute the two independent solutions of the homogeneous BPT Eq. (3.15), $\Psi_{\ell}^{\mathrm{in} / \mathrm{out}}\left(r_{i}, \omega_{j}\right)$. Finally, the amplitude of the emitted gravitational wave can be computed by numerically evaluating the integrals (3.13) and (3.19), which then yield the energy spectra (3.14) and (3.21).

With the exception of the handling of the solution of the relativistic hydrodynamical equations, the procedure described so far for the combination of the time and frequency-domain approaches is straightforward and with minimal computational requirements. However, great attention needs to be paid in to avoid unphysical results. In particular, even when the timeseries extend over several tens of dynamical timescales and the time sampling is also very high, the calculation of the Fourier transforms can be inaccurate and this can be problematic particularly at very large frequencies. Indeed, we have found that the energy spectrum can become divergent at frequencies above a few $\mathrm{kHz}$ even when the hydrodynamical evolution is carried out over $\sim 100$ dynamical timescales.

The reason for this is most easily seen within the Green's function approach; in this case, in fact, when $\omega$ increases above a few $\mathrm{kHz}$, the Wronskian of the homogeneous solutions tends to zero very rapidly. At least in principle, 
this rapid decay should be compensated by an equivalent decrease of the source function $S_{\ell}^{( \pm)}$or $S_{\ell}^{\mathrm{BPT}}$ such that the wave amplitude would remain finite. In practice, however, this is not necessarily the case and if the decay in the source functions is not sufficiently rapid, because for instance their values at high frequencies are not sufficiently accurate, this would inevitably lead to the high-frequency divergences we have observed. Fortunately, a simple solution to this otherwise serious problem is possible. In the limit of an infinite time integration interval, in fact, it is possible to replace the Fourier transform at a given frequency $\omega$ of a timeseries $h(t)$ with the Fourier transform of its time derivative divided by $\omega$, i.e.

$$
h(\omega)=\frac{\mathrm{i}}{\omega}\left(\frac{1}{2 \pi} \int_{-\infty}^{\infty} \frac{d h(t)}{d t} e^{\mathrm{i} \omega t} d t\right),
$$

This identity, which is strictly true if $h(t)=0$ for $t \rightarrow \pm \infty$, can be exploited to compensate the loss of accuracy in the Fourier transform. As a result, in our approach, and before Fourier transforming the source terms $A_{\ell}, A_{\ell}^{(1)}, \ldots$ in (3.23), we compute their first and second time derivatives, evaluate the corresponding Fourier transform and finally divide the result by $\omega^{2}$. This method effectively removes the divergence appearing at large frequencies and has proven to work well also in the case in which the timeseries is, in practice, finite.

\section{A REPRESENTATIVE EXAMPLE: AN OSCILLATING HIGH-DENSITY TORUS}

The procedure discussed in the previous two Sections is totally generic and could in principle be used to investigate the perturbations induced on a Schwarzschild spacetime by an extended matter source when the latter dynamics is simulated consistently in more than one spatial dimension. However, as mentioned in the Introduction, we will here concentrate on a proof of its effectiveness by considering the perturbations induced by a nonselfgravitating torus orbiting and oscillating around a Schwarzschild black hole. The latter will be simulated with the 2D general relativistic code discussed in Sec. III A.

In order to construct the background initial model for the torus, which we subsequently perturb, we consider a perfect fluid described by the stress-energy tensor (3.1) and in circular nongeodesic motion with four-velocity $u^{\alpha}=$ $\left(u^{t}, 0,0, u^{\phi}\right)=u^{t}(1,0,0, \Omega), \quad$ where $\quad \Omega=\Omega(r, \theta) \equiv$ $u^{\phi} / u^{t}$ is the coordinate angular velocity as observed from infinity. Enforcing the conditions of hydrostatic equilibrium and of axisymmetry simplifies the hydrodynamics equations considerably and for a nonselfgravitating fluid these reduce to Bernoulli-type equations

$$
\frac{\nabla_{i} p}{e+p}=-\nabla_{i} W+\frac{\Omega \nabla_{i} l}{1-\Omega l}
$$

where $i=r, \theta, W \equiv \ln \left(u_{t}\right)$ and $l \equiv-u_{\phi} / u_{t}$ is the specific angular momentum.

The simplest solution to Eqs. (4.1) is the one with $l=$ const., since in this case the equipotential surfaces can be computed directly through the metric coefficients and the value of the specific angular momentum. Note that at any point in the $(r, \theta)$ plane, the potential $W$ can either be positive (indicating equipotential surfaces that are open) or negative (indicating equipotential surfaces that are closed). The case $W=0$ refers to that special equipotential surface which is closed at infinity (see Refs. [17,18] for details). local extrema on the equatorial plane of closed equipotential surfaces mark the radial positions of the cusp, $r_{\text {cusp }}$, and of the " center" of the torus, $r_{\mathrm{c}}$. At these radial positions the specific angular momentum must be that of a Keplerian geodesic circular orbit which can effectively be used to calculate the position of both the center and the cusp.

Stationary solutions with constant specific angular momentum are particularly useful since in this case the angular velocity is fully determined as $\Omega=l g_{t t} / g_{\phi \phi}$ and if a polytropic EOS is used, the Bernoulli Eqs. (4.1) can be integrated analytically to yield the rest-mass density (and pressure) distribution inside the torus as

$$
\rho(r, \theta)=\left\{\frac{\gamma-1}{\kappa \gamma}\left[\exp \left(W_{\text {in }}-W\right)-1\right]\right\}^{1 /(\gamma-1)},
$$

where $W_{\text {in }} \equiv W\left(r_{\text {in }}, \pi / 2\right)$. Clearly, different configurations can be built depending on the value chosen for $l$, with finite-extent tori resulting when $l_{\mathrm{ms}}<l<l_{\mathrm{mb}}$, with $l_{\mathrm{mb}}=$ $4, l_{\mathrm{ms}}=3 \sqrt{3 / 2}$ being the specific angular momenta corresponding to orbits that are marginally bound or marginally stable, respectively.

The configurations built in this way have a large quadrupole moment but being stationary they do not produce any time-varying perturbation to the background spacetime. Because of this we introduce parametrized perturbations that would induce a small outflow through the cusp and excite a quasiharmonic behavior in the hydrodynamical variables (the interested reader is referred to Refs. [2629] for a detailed discussion of the harmonic properties of this type of oscillations). More specifically, we modify the stationary equilibrium configuration with a small radial velocity which we have expressed in terms of the radial inflow velocity characterizing a relativistic spherically symmetric accretion flow onto a Schwarzschild black hole, i.e., the Michel solution [30]. Using $\eta$ to parameterize the strength of the perturbation, we have specified the initial radial (covariant) component of the three-velocity as

$$
v_{r}=\eta\left(v_{r}\right)_{\text {Michel }} \text {. }
$$

As a result of the introduction of the initial perturbation, the torus acquires a linear momentum in the radial direction pushing it towards the black hole. When this happens, 

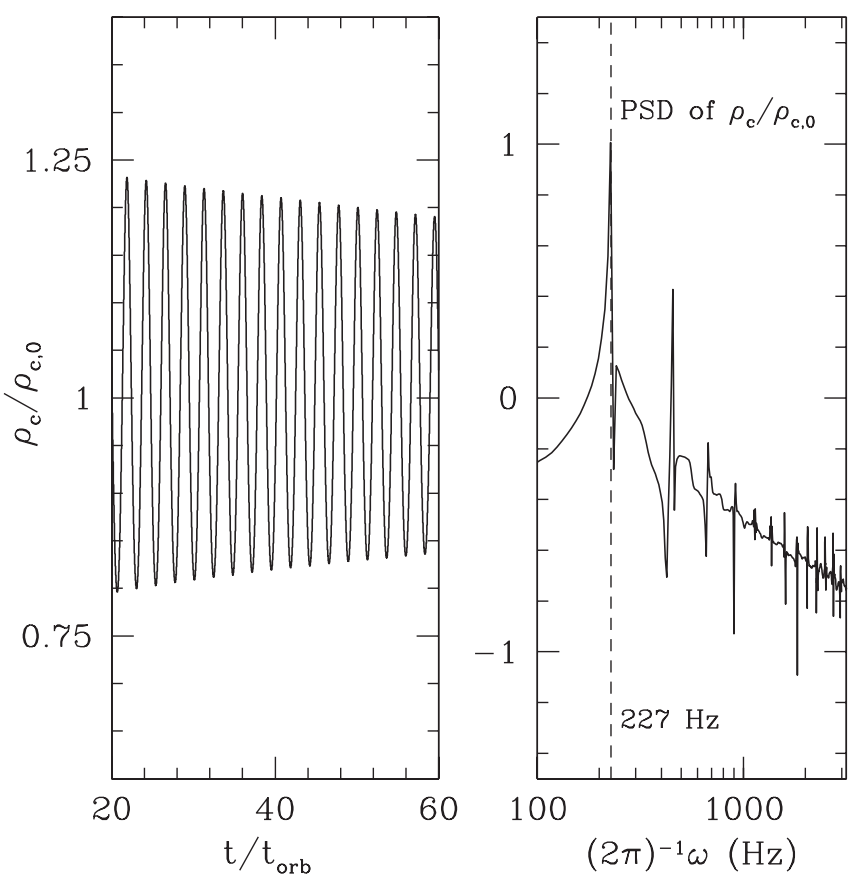

FIG. 1. Harmonic behavior of an oscillating torus. Both panels refer to model (c) of Table I which was perturbed with a radial velocity having $\eta=0.05$. The left panel shows the time variation of the maximum rest-mass density normalized to its initial value for a representative portion of the timeseries. The right panel shows the power spectral density (PSD) calculated over 100 orbital timescales, with the eigenfrequency of the $f$ mode indicated with a vertical dashed line at $227 \mathrm{~Hz}$.

the pressure gradients become stronger to counteract the steeper gravitational potential experienced as the torus moves inward, thus increasing the central density and eventually pushing the torus back to its original position. The resulting oscillations are essentially harmonic in the fundamental $f$-mode of oscillation but other, higher-order $p$ modes are also excited and appear at frequencies that are in a ratio of small integers [26-29]. This is summarized in the two panels of Fig. 1 which refer to model (c) of Table I which was perturbed with a radial velocity having $\eta=$ 0.05 and evolved for several tens of the orbital timescale $t_{\mathrm{orb}}=1.86 \mathrm{~ms}$. The left panel shows as a function of time the variation of the maximum rest-mass density (i.e., the rest-mass density at the center of the torus $\rho_{c}$ ) normalized to its initial value for a representative portion of the timeseries. The right panel, on the other hand, shows as a function of the frequency $\nu \equiv \omega /(2 \pi)$, the power spectral density (PSD) in arbitrary units of the timeseries calculated over about 100 orbital timescales. Indicated with a vertical dashed line at $\bar{\nu}=227 \mathrm{~Hz}$ is the eigenfrequency of the fundamental $(f)$ mode of oscillation and smaller peaks at integer and semi-integer multiples of $\bar{\nu}$ are also visible and are referred to the first overtones (see Ref. [29] for a discussion of these modes).

As the simulation is carried out and the hydrodynamical equations are solved along the lines discussed in Sec. III A, the source functions (3.23) are calculated at the different radial gridpoints and stored as distinct output. Once the dynamics has been followed for a sufficient time-span comprising several tens orbital timescales, the simulation in the time domain is stopped and the values of source functions (3.23) throughout the simulated spacetime are read-in at the different radial positions. This marks the begin of the analysis in the frequency domain, which first produces the source functions (3.24) and then proceeds to the solution of the perturbation Eqs. (2.2) and (2.8) along the lines discussed in Sec. III B. The results of the hybrid approach are then summarized in Figs. 2-4 which show the energy spectrum of the emitted gravitational radiation as a function of the frequency.

The first figure, in particular, has been computed within the Newtonian quadrupole formalism through expression (2.13) and being a faithful mirror of the hydrodynamical quantities, it shows a main peak at $227 \mathrm{~Hz}$ as well as the smaller peaks already discussed for the right panel of Fig. 1. Note that despite the use of the procedure described at the end of Sec. IIIC, the very steep dependence on the frequency as $\omega^{6}$ of the energy spectrum [cf. Eq. (2.13)], produces an incorrect but finite growth at high frequencies, where the accuracy in the calculation of the sources is smaller. Of course, being rooted in a Newtonian approximation, the energy spectrum in Fig. 1 shows no sign of a contribution coming from the QNMs of the black hole that, for a mass of $M=2.5 M_{\odot}$ are expected at $\nu=4.828 \mathrm{kHz}$ for the the fundamental $\ell=2-\mathrm{QNM}$.

TABLE I. Main properties of the constant angular momentum toroidal neutron star models used in the numerical calculations. From left to right the columns report: the name of the model, the star-to-hole mass ratio $M_{\mathrm{t}} / M$, the polytropic constant $\kappa$, the specific angular momentum $\ell$ (normalized to $M$ ), the inner and outer radii of the toroidal neutron star $r_{\text {in }}$ and $r_{\text {out }}$, the radial position of cusp $r_{\text {cusp }}$, the

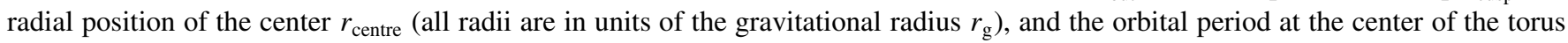
$t_{\text {orb }}$, expressed in milliseconds. The last two columns indicate the density at the center of the torus and the average density of each model, respectively, both in cgs units. All of the models share the same mass for the black hole, $M=2.5 M_{\odot}$ and adiabatic index $\gamma=4 / 3$.

\begin{tabular}{|c|c|c|c|c|c|c|c|c|c|c|}
\hline Model & $M_{\mathrm{t}} / M$ & $\kappa(\mathrm{cgs})$ & $\ell$ & $r_{\text {in }}$ & $r_{\text {out }}$ & $r_{\text {cusp }}$ & $r_{\text {centre }}$ & $t_{\mathrm{orb}}(\mathrm{ms})$ & $\rho_{\text {centre }}(\operatorname{cgs})$ & $\langle\rho\rangle(\mathrm{cgs})$ \\
\hline (c) & 0.1 & $0.96 \times 10^{14}$ & 3.8000 & 4.576 & 15.889 & 4.576 & 8.352 & 1.86 & $1.14 \times 10^{13}$ & $4.73 \times 10^{11}$ \\
\hline (e) & 0.1 & $7.0 \times 10^{13}$ & 3.7845 & 4.646 & 14.367 & 4.646 & 8.165 & 1.80 & $1.61 \times 10^{13}$ & $6.43 \times 10^{11}$ \\
\hline (f) & 0.1 & $1.0 \times 10^{14}$ & 3.8022 & 4.566 & 16.122 & 4.566 & 8.378 & 1.87 & $1.10 \times 10^{13}$ & $4.48 \times 10^{11}$ \\
\hline
\end{tabular}




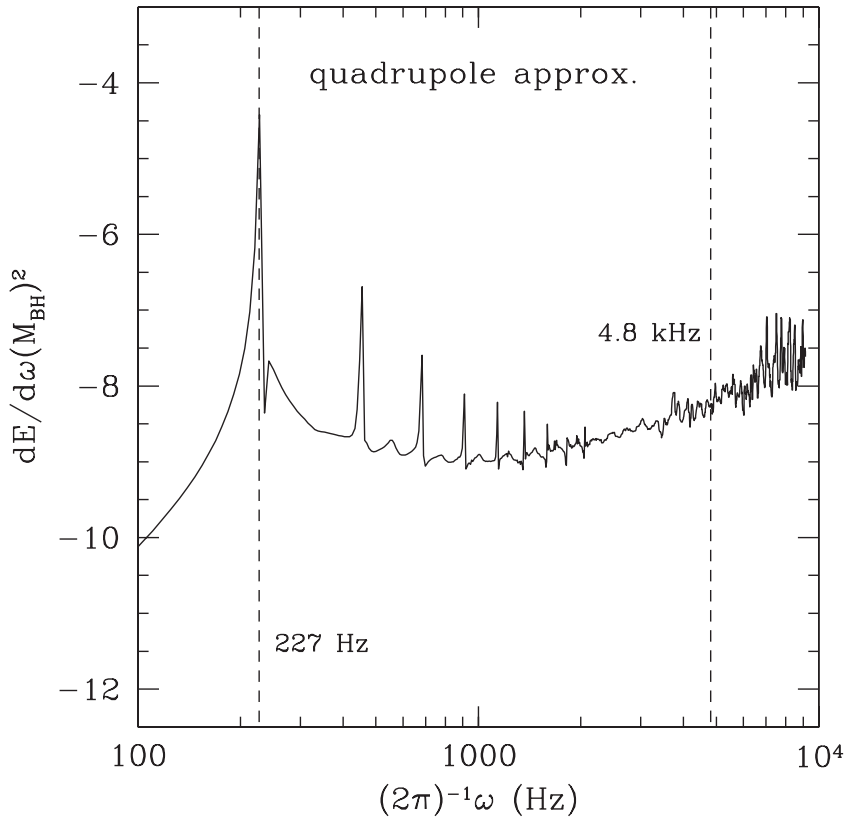

FIG. 2. Gravitational-wave energy spectrum produced by an oscillating, high-density torus orbiting around a $2.5 M_{\odot}$ Schwarzschild black hole. The radiation has been computed for the $\ell=2$ mode using Eq. (2.13) within the Newtonian quadrupole approximation.

Figure 3 shows instead the equivalent gravitationalwave energy spectrum resulting from the hybrid approach employing the solution of the RWZ equations [cf. Eq. (2.6)] for $\ell=2$. A number of interesting features should be noted. Firstly, at low frequencies the spectra derived through the perturbative approach shows a rather

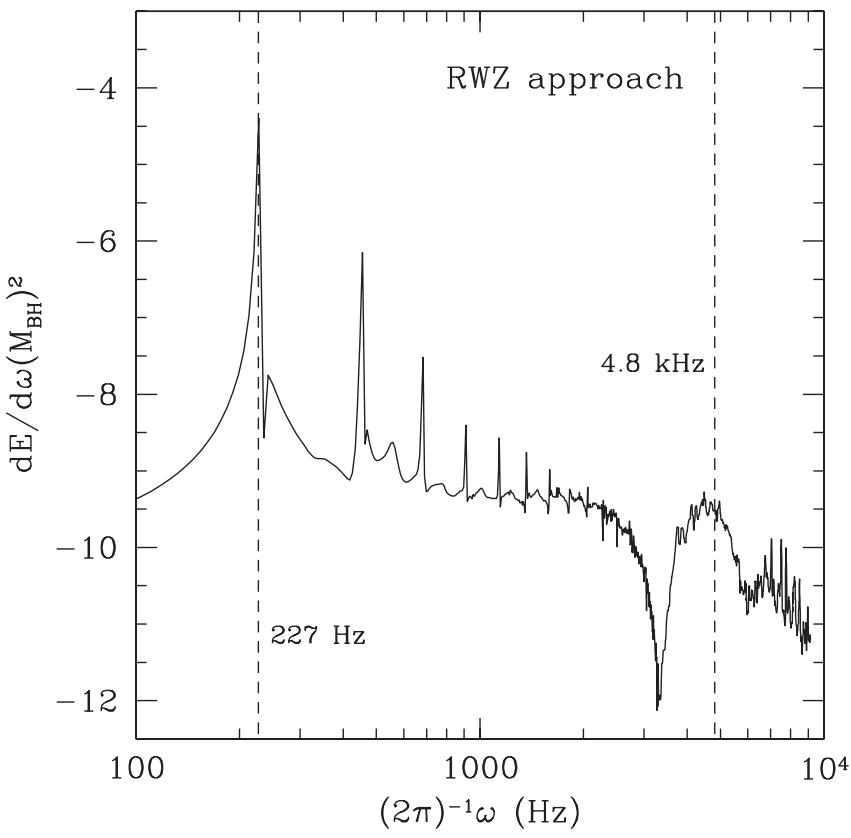

FIG. 3. Same as Fig. 2 but derived integrating the RWZ equations in the frequency domain for $\ell=2$ and using Eq. (2.6).

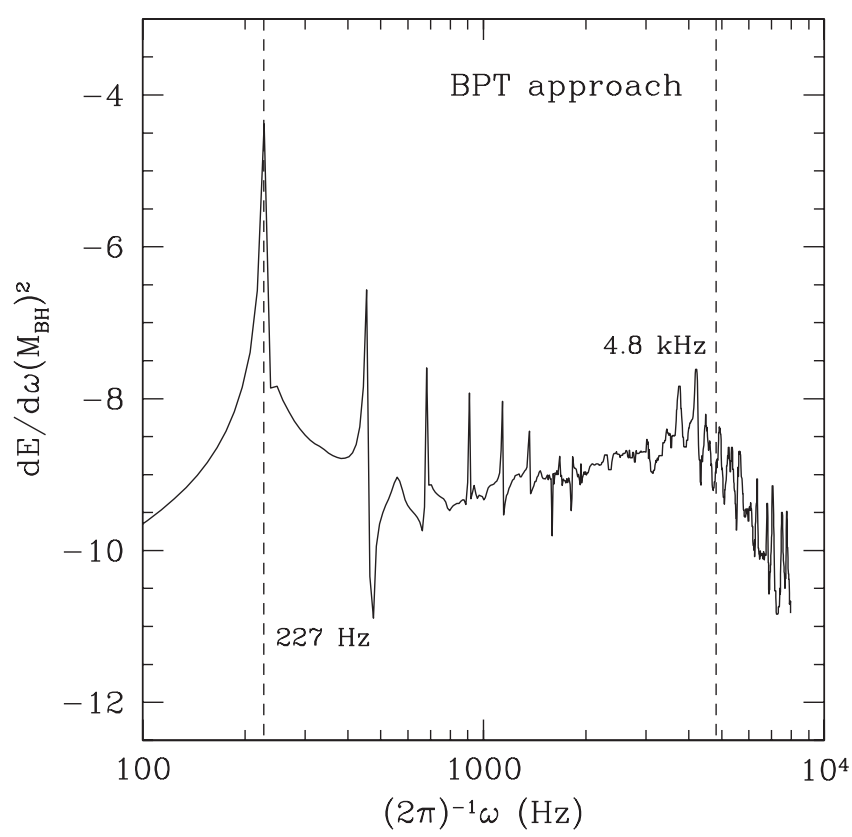

FIG. 4. Same as Fig. 2 but obtained integrating the BPT equation in the frequency domain for $\ell=2$ and using Eq. (2.11).

good agreement with the quadrupole spectrum given in Fig. 2. This confirms what already noted in [13] and that, at least for this type of sources, the quadrupole approach captures the most important qualitative features of the energy spectrum. Secondly, the spectrum is not diverging, nor growing at large frequencies as a result of the use of the transformation (3.25). Finally and most importantly, the spectrum is not monotonically decreasing but shows a distinctive and broad peak at $\nu \sim 4.4 \mathrm{kHz}$, rather close to the position of the expected fundamental $\ell=2$ QNM of the black hole and which we interpret as the excitation of the QNMs of the black hole resulting from the perturbations induced by the oscillating torus. Although its contribution is energetically very small (i.e. less that $2 \times 10^{-4}$ of the total emitted energy is produced at frequencies larger than $2 \mathrm{kHz}$ ), the ability to isolate this peak is of great importance to assess the validity of the hybrid approach and to prove its effectiveness in modelling black hole spacetimes. The presence of such a peak, in fact, is expected on the basis of simple considerations but was not detectable in the complementary work of Nagar et al. [13] based on a time-domain perturbative approach despite the corresponding PSD reached values was well below the ones at which our black hole peak appears (cf. Fig. 3 with the left panel of Fig. 1 in Ref. [13]).

Finally, we show in Fig. 4 the gravitational-wave energy spectrum resulting from the hybrid approach employing the solution of the BPT equation [cf. Eq. (2.11)] for $\ell=2$. As expected, much of what found for the RWZ equations continues to hold when the hybrid approach is computed using the BPT equation, namely: very good match with the quadrupole approximation at low frequency where most of 
the energy is emitted and the appearance of a broad peak associated with the black hole quasinormal ringing.

\section{CONCLUSIONS}

We have presented a new hybrid approach to study the perturbations of a Schwarzschild black hole excited by extended matter sources in which the solution in the time domain of the relativistic hydrodynamical equations in a multidimensional spacetime is coupled to the solution in the frequency domain of either the Regge-Wheeler/Zerilli equations or of the Bardeen-Press-Teukolsky equations.

We believe that our hybrid approach may be preferrable over a pure time-domain approach based on the RWZ equations for at least three different reasons. Firstly, the Regge-Wheeler and the Zerilli equations cannot be generalized to rotating black holes, whereas the BPT equation admits such a generalization. Secondly, the BPT equation is intrinsically unstable when integrated in the time domain but its solution is regular computed in the frequency domain. Thirdly, for a system undergoing small oscillations around an equilibrium state, a frequency-domain approach is expected to be significantly more accurate than one based in the time domain, especially at high frequencies, where the black hole contributions are expected to emerge. Within this framework, therefore, the work presented here serves as a first step towards the study of the gravitationalwave emission from an extended source perturbing a rotating black hole.

As a test of its effectiveness and to provide a close comparison with what has been done so far in perturbative approaches based in the time domain, i.e., Ref. [13], we have considered as extended source to the perturbation equations the oscillations of a high-density torus orbiting around a Schwarzschild black hole. The dynamics of the torus has been followed numerically using a 2D numerical code solving the general relativistic hydrodynamics equations using HRSC schemes in spherical polar coordinates.

Overall, the results of our study show that the hybrid approach (either with the RWZ equations or with the BPT one) works remarkably well, producing an energy spectrum of the emitted gravitational radiation which contains both the basic quadrupolar emission of the torus as well as the excited emission of the black hole. The latter feature was completely washed out in the complementary timedomain approach of Ref. [13].

Being totally generic, the formalism and methodology presented here is could be used under more general conditions and, in particular, also with sources coming from 3D numerical relativity codes as long as an accurate evolution of the matter sources is possible. Future work in this line of research will therefore comprise both the extension of the present results to 3D codes, as well as the generalization of the perturbative formalism to the Teukolsky equation in order to model the gravitational emission of rotating black holes when perturbed by extended sources.

\section{ACKNOWLEDGMENTS}

It is a pleasure to thank J. A. Font, A. Nagar, J. A. Pons and O. Zanotti for useful comments and discussions. Support to this research comes also through the SFBTR7 "Gravitationswellenastronomie" of the DFG and the Italian INFN, Iniziativa Specifica OG51. The computations were performed on the Beowulf Cluster for numerical relativity "Albert" at the University of Parma.

\section{APPENDIX A: GENERIC SOURCE TERMS}

\section{Source for the RWZ equations}

The source of the RWZ Eqs. (2.2), $S_{\ell}^{( \pm)}(r, \omega)$, can be expressed in terms of the components of the stress-energy tensor $T_{\mu \nu}(t, r, \theta, \phi)$ as follows. We first decompose $T_{\mu \nu}$ in tensor spherical harmonics for the elevant components

$$
\begin{gathered}
T_{t r}(r, t)=\frac{\mathrm{i}}{\sqrt{2}} \sum_{\ell m} \tilde{A}_{\ell m}^{(1)} Y^{\ell m}, \\
T_{r r}(r, t)=\sum_{\ell m} \tilde{A}_{\ell m} Y^{\ell m}, \\
T_{t a}(r, t)=\frac{\mathrm{i} r}{\sqrt{2 \Lambda^{\prime}}} \sum_{\ell m}\left(\tilde{B}_{\ell m}^{(0)} Y_{, a}^{\ell m}-\tilde{Q}_{\ell m}^{(0)} S_{a}^{\ell m}\right), \\
T_{r a}(r, t)=\frac{r}{\sqrt{2 \Lambda^{\prime}}} \sum_{\ell m}\left(\tilde{B}_{\ell m} Y^{\ell m}{ }_{, a}-\tilde{Q}_{\ell m} S_{a}^{\ell m}\right), \\
T_{a b}(r, t)=\frac{r^{2}}{\sqrt{2}} \sum_{\ell m}\left(\tilde{G}_{\ell m} Y^{\ell m} \gamma_{a b}+\frac{\tilde{F}_{\ell m} Z_{a b}^{\ell m}-\mathrm{i} \tilde{D}_{\ell m} S_{a b}^{\ell m}}{\sqrt{2 \Lambda \Lambda^{\prime}}}\right),
\end{gathered}
$$

where $a=\theta, \phi, \gamma_{a b} \equiv \operatorname{diag}\left(1, \sin ^{2} \theta\right)$ is the metric tensor of the sphere, $Y^{\ell m}$ are the scalar spherical harmonics, $Y^{\ell m}{ }_{, a} \equiv \partial Y^{\ell m} / \partial x^{a} \quad$ and $\quad \Lambda \equiv(\ell-1)(\ell+2) / 2, \quad \Lambda^{\prime} \equiv$ $\ell(\ell+1)$. The matrices

$$
\begin{gathered}
S_{a}^{\ell m}=\left(-Y_{, \phi}^{\ell m} / \sin \theta, \sin \theta Y_{, \theta}^{\ell m}\right), \\
Z_{a b}^{\ell m}=\left(\begin{array}{cc}
W^{\ell m} & X^{\ell m}, \\
X^{\ell m} & -\sin ^{2} \theta W^{\ell m}
\end{array}\right), \\
S_{a b}^{\ell m}=\left(\begin{array}{cc}
-X^{\ell m} / \sin \theta & \sin \theta W^{\ell m} \\
\sin \theta W^{\ell m} & \sin \theta X^{\ell m}
\end{array}\right),
\end{gathered}
$$

with

$$
\begin{gathered}
W^{\ell m}=Y_{, \theta \theta}^{\ell m}-\cot \theta Y_{, \theta}^{\ell m}+\frac{m^{2}}{\sin ^{2} \theta} Y^{\ell m}, \\
X^{\ell m}=2 \mathrm{i} m\left(Y_{, \theta}^{\ell m}-\cot \theta Y^{\ell m}\right),
\end{gathered}
$$

are vector and tensor spherical harmonics, and collect all of the angular dependence. 
Next, using the orthogonality properties of $Y^{\ell m}, S_{a}^{\ell m}$, $S_{a b}^{\ell m}, Z_{a b}^{\ell m}$ and the axial symmetry of the stress-energy tensor it is possible to show that only the $m=0$ harmonics contribute to the expansion (A5) and that

$$
\begin{gathered}
\tilde{A}_{\ell}=2 \pi \int d \theta \sin \theta Y^{\ell 0} T_{r r}, \\
\tilde{A}_{\ell}^{(1)}=-\mathrm{i} \sqrt{2} 2 \pi \int d \theta \sin \theta Y^{\ell 0} T_{t r} \\
\tilde{B}_{\ell}^{(0)}=-\mathrm{i} \lambda_{1} 2 \pi \int d \theta \sin \theta T_{t \theta} Y^{\ell 0}{ }_{, \theta}, \\
\tilde{Q}_{\ell}=\lambda_{1} 2 \pi \int d \theta \sin \theta \frac{1}{\sin \theta} T_{r \phi} Y^{\ell 0}{ }_{, \theta}, \\
\tilde{F}_{\ell}=\lambda_{2} 2 \pi \int d \theta \sin \theta T_{r \theta} Y^{\ell 0}, \\
\tilde{D}_{\ell}=-\mathrm{i} \lambda_{2} 2 \pi \int d \theta \sin \theta\left(T_{\theta \theta}-\frac{1}{\sin { }^{2} \theta} T_{\phi \phi}\right) W^{\ell 0}, \\
\tilde{B}_{\ell} \theta\left(-\frac{2}{\sin \theta} T_{\theta \phi}\right) W^{\ell 0},
\end{gathered}
$$

where

$$
\begin{gathered}
\lambda_{1} \equiv \frac{\sqrt{2}}{r \sqrt{\Lambda^{\prime}}}, \\
\lambda_{2} \equiv \frac{1}{r^{2} \sqrt{4 \Lambda \Lambda^{\prime}}} .
\end{gathered}
$$

In terms of the Fourier transform of these quantities, the source terms $S_{\ell}^{( \pm)}(r, \omega)$ are

$$
\begin{aligned}
S^{(+)}(r, \omega)= & \alpha_{0} A^{(1)}+\alpha_{1} A_{r}^{(1)}+\beta A+\gamma B+\delta F+\epsilon_{2} B_{, r r}^{(0)} \\
& +\epsilon_{1} B_{, r}^{(0)}+\epsilon_{0} B^{(0)}, \\
& S^{(-)}(r, \omega)=\sigma_{1}\left(-g_{00} D\right)_{, r}+\chi Q,
\end{aligned}
$$

with

$$
\begin{gathered}
\alpha_{0}=4 \pi \sqrt{2} \frac{M(r-2 M)((\Lambda+3) r-3 M)}{r(\Lambda r+3 M)^{2} \omega}, \\
\alpha_{1}=2 \pi \sqrt{2} \frac{(r-2 M)^{2}}{\omega(\Lambda r+3 M)}, \\
\beta=4 \pi \frac{(r-2 M)^{2}}{\Lambda r+3 M}, \\
\gamma=4 \pi \sqrt{\frac{2}{\Lambda^{\prime}}} \frac{(r-2 M)^{2}}{\Lambda r+3 M}, \\
\delta=-\frac{8 \pi}{\sqrt{\Lambda \Lambda^{\prime}}}(r-2 M),
\end{gathered}
$$

$$
\begin{gathered}
\epsilon_{2}=-4 \pi \sqrt{\frac{2}{\Lambda^{\prime}}} \frac{r(r-2 M)^{2}}{\omega(\Lambda r+3 M)}, \\
\epsilon_{1}=-4 \pi \sqrt{\frac{2}{\Lambda^{\prime}}} \frac{(r-2 M)\left(3 \Lambda r^{2}+15 M r-4 M \Lambda r-24 M^{2}\right)}{\omega(\Lambda r+3 M)^{2}}, \\
\epsilon_{0}=4 \pi \sqrt{\frac{2}{\Lambda^{\prime}} \frac{1}{\omega}} \\
\times\left[\frac{(r-2 M)\left(\Lambda^{2} r^{2}-3 M \Lambda r-12 M r+12 M^{2}\right)}{r(\Lambda r+3 M)^{2}}\right. \\
\left.-\frac{\omega^{2} r^{3}}{\Lambda r+3 M}\right], \quad \\
\chi=-8 \pi \mathrm{i} \sqrt{\frac{2}{\Lambda^{\prime}}} \frac{(r-2 M)^{2}}{r^{2}}, \\
\sigma_{1}=\mathrm{i} \frac{8 \pi}{\sqrt{\Lambda \Lambda^{\prime}}}(r-2 M) .
\end{gathered}
$$

\section{Source for the BPT equation}

The source of the BPT Eq. (2.8), $S_{\ell}^{\mathrm{BPT}}(r, \omega)$, can be expressed in terms of the components of the stress-energy tensor as follows (see $[15,31])$

$$
\begin{aligned}
S_{\ell}^{\mathrm{BPT}}(r, \omega)= & -\sqrt{2 \Lambda \Lambda^{\prime}} r^{4} T_{(n)(n) \ell} \\
& -\sqrt{2 \Lambda^{\prime}} \Delta D_{+}\left[\frac{r^{5}}{\Delta} T_{(n)(\bar{m}) \ell]}\right] \\
& -\frac{\Delta}{2 r} D_{+}\left[\frac{r^{6}}{\Delta} D_{+}\left(r T_{(\bar{m})(\bar{m}) \ell}\right)\right],
\end{aligned}
$$

where $D_{+} \equiv d / d r_{*}+i \omega$, and $T_{(n)(n) \ell}, T_{(n)(\bar{m}) \ell}, T_{(\bar{m})(\bar{m}) \ell}$ are the Fourier transform of the following quantities

$$
\begin{aligned}
\tilde{T}_{(n)(n) \ell} & \equiv \int d \Omega_{0} S_{\ell 0}(\theta) T^{\mu \nu} n_{\mu} n_{\nu}, \\
\tilde{T}_{(n)(\bar{m}) \ell} & \equiv \int d \Omega_{-1} S_{\ell 0}(\theta) T^{\mu \nu} n_{\mu} \bar{m}_{\nu}, \\
\tilde{T}_{(\bar{m})(\bar{m}) \ell} & \equiv \int d \Omega_{-2} S_{\ell 0}(\theta) T^{\mu \nu} \bar{m}_{\mu} \bar{m}_{\nu},
\end{aligned}
$$

where $d \Omega=\sin \theta d \theta d \phi$ is the solid angle and the oneforms $n_{\mu}, \bar{m}_{\mu}$ are defined as

$$
\begin{aligned}
n_{\mu} & \equiv \frac{1}{2}\left(1-\frac{2 M}{r}, 1,0,0\right), \\
\bar{m}_{\mu} & \equiv-\frac{1}{\sqrt{2}}(0,0, r,-\mathrm{i} r \sin \theta) .
\end{aligned}
$$

Here, ${ }_{-s} S_{\ell m}(\theta, \phi)$ are the spin-weighted spherical harmonics and we have considered only those with $m=0$ because of the underlying axisymmetry in the perturbations. 
[1] T. Regge and J. A. Wheeler, Phys. Rev. 108, 1063 (1957).

[2] F. Zerilli, Phys. Rev. D 2, 2141 (1970).

[3] A. Nagar and L. Rezzolla, Classical Quantum Gravity 22, R167 (2005).

[4] S. A. Teukolsky, Phys. Rev. Lett. 29, 1114 (1972).

[5] T. Nakamura, K. Oohara, and Y. Kojima, Prog. Theor. Phys. Suppl. 90, 1 (1987).

[6] C. Cutler, D. Kennifick, and E. Poisson, Phys. Rev. D 50, 3816 (1994).

[7] L. Gualtieri, E. Berti, J.A. Pons, G. Miniutti, and V. Ferrari, Phys. Rev. D 64, 104007 (2001).

[8] J. A. Pons, E. Berti, L. Gualtieri, G. Miniutti, and V. Ferrari, Phys. Rev. D 65, 104021 (2002).

[9] S. L. Detweiler and E. Szedenits, Astrophys. J. 231, 211 (1979).

[10] M. P. Haugan, S. L. Shapiro, and I. Wasserman, Astrophys. J. 257, 283 (1982).

[11] S. L. Shapiro and I. Wasserman, Astrophys. J. 260, 838 (1982); Mon. Not. R. Astron. Soc. 344, 978 ( 2003).

[12] P. Papadopoulos and J. A. Font, Phys. Rev. D 59, 044014 (1999).

[13] A. Nagar, J. A. Font, O. Zanotti, and R. De Pietri, Phys. Rev. D 72, 024007 (2005).

[14] J. M. Bardeen and W. H. Press, J. Math. Phys. (N.Y.) 14, 7 (1973).

[15] S. A. Teukolsky, Astrophys. J. 185, 635 (1973).

[16] S. E. Woosley, Astrophys. J. 405, 273 (1993).

[17] J. A. Font and F. Daigne, Mon. Not. R. Astron. Soc. 334,
383 (2002).

[18] O. Zanotti, L. Rezzolla, and J. A. Font, Mon. Not. R. Astron. Soc. 341, 832 (2003).

[19] M. Shibata and K. Taniguchi, Phys. Rev. D 73, 064027 (2006).

[20] M. D. Duez, Y. T. Liu, S. L. Shapiro, M. Shibata, and B. C. Stephens, Phys. Rev. Lett. 96, 031101 (2006).

[21] M. Vietri and L. Stella, Astrophys. J. 507, L45 (1998).

[22] A. I. MacFayden and S. E. Woosley, Astrophys. J. 524, 262 (1999).

[23] L. Baiotti, I. Hawke, P. J. Montero, L. Löffler, L. Rezzolla, N. Stergioulas, J. A. Font, and E. Seidel, Phys. Rev. D 71, 024035 (2005).

[24] M. Davis, R. Ruffini, W. H. Press, and R. H. Price, Phys. Rev. Lett. 27, 1466 (1971).

[25] S. Chandrasekhar, The Mathematical Theory of Black Holes (Clarendon Press, Oxford, 1984), Chap. 4, Sec. 26.

[26] O. Zanotti, J. A. Font, L. Rezzolla, and P. J. Montero, Mon. Not. R. Astron. Soc. 356, 1371 (2005).

[27] P. J. Montero, L. Rezzolla, and S'i. Yoshida, Mon. Not. R. Astron. Soc. 354, 1040 (2004).

[28] L. Rezzolla, T. W. Maccarone, S'i. Yoshida, and O. Zanotti, Mon. Not. R. Astron. Soc. 344, L37 (2003).

[29] L. Rezzolla, S'i. Yoshida, and O. Zanotti, Mon. Not. R. Astron. Soc. 344, 978 (2003).

[30] F. Michel, Astrophys. Space Sci. 15, 153 (1972

[31] M. Sasaki and T. Nakamura, Phys. Lett. A 87, 85 (1981). 